\title{
Aborto no Brasil: uma pesquisa domiciliar com técnica de urna
}

\author{
Abortion in Brazil: a household survey \\ using the ballot box technique
}

Debora Diniz ${ }^{1}$

$M$ arcelo $M$ edeiros ${ }^{2}$

\footnotetext{
${ }^{1}$ Anis-Instituto de Bioética, Direitos Humanos e Gênero. Caixa Postal 8011, Setor Sudoeste. 70673-970 Brasília DF. anis@anis.org.br ${ }^{2} U n B$.
}

Abstract This study presents the first results of the National Abortion Survey (PNA, Pesquisa Nacional deAborto), a household random sample survey fielded in 2010 covering urban women in Brazil aged 18 to 39 years. The PNA combined two techniques, interviewer-administered questionnaires and self-administered ballot box questionnaires. The results of PNA show that at the end of their reproductive health one in five women has performed an abortion, with abortions be ing more frequent in the main reproductive ages, that is, from 18 to 29 years old. No relevant differentiation was observed in the practice of abortion among religious groups, but abortion was found to be more common among people with lower education. The use of medical drugs to induce abortion occurred in half of the abortions, and postabortion hospitalization was observed among approximately half of the women who aborted. Such results lead to conclude that abortion is a priority in the Brazilian public health agenda.

Key words Induced abortion, Abortion surveys, M edical abortion, Reproductivehistory, Ballot box technique, Brazil
Resumo 0 artigo apresenta os primeiros resultados da Pesquisa Nacional deAborto (PNA), um levantamento por amostragem aleatória de domićlios, realizado em 2010, cuja cobertura abrangeu as mulheres com idades entre 18 e 39 anos em todo o Brasil urbano. A PN A combinou duas técnicas de sondagem: a técnica deurna equestionários preenchidos por entrevistadoras. Seus resultados indicam que, ao final da vida reprodutiva, mais de uma em cada cinco mulheres já fez aborto, ocorrendo os abortos em geral nas idades que compõem o centro do período reprodutivo das mulheres, isto é, entre 18 e 29 anos. Não se observou diferenciação relevante na prática em função de crença religi osa, mas o aborto se mostrou mais comum entre mulheres de menor escolaridade. 0 uso de medicamentos para a indução do último aborto ocorreu em metade dos casos e a internação pós-aborto foi observada em cerca de metade dos abortos. Tais resultados levam a concluir que 0 aborto deve ser prioridade na agenda de saúde pública nacional.

Palavras-chave Aborto induzido, Pesquisa de aborto, Aborto médico, H istória reprodutiva, T écnica de urna, Brasil 
Introdução

Os dados sobre a magnitude do aborto provocado no Brasil devem ser examinados à luz do contexto restritivo da lei. 0 aborto é um crime e as mulheres são penalizadas por sua prática. Os pesquisadores não têm como oferecer medidas de sigilo ou proteção às mulheres que participarem das pesquisas, sejam elas realizadas em hospitais ou em suas residências. $N$ ão há direito ao sigilo para o exercício da pesquisa no Brasil. Foi nesse contexto paradoxal decontrasteentrealei penal e as necessidades de saúde das mulheres que grande parte dos estudos sobre magnitude do aborto foi conduzida no Brasil nas últimas décadas para subsidiar as políticas de saúde reprodutiva ${ }^{1,2}$.

Os principais estudos sobre a magnitude do aborto no Brasil utilizaram três tipos de abordagem metodológica ${ }^{1}$. 0 primeiro parte de registros de internações hospitalares para procedimentos médicos relacionados à prática do aborto, tais como a curetagem, sendo os cálculos mais recentes baseados em registros do Sistema Ú nico de Saúde (SUS). A estes registros de internação, aplicam-se fatores de correção para estimar o fenômeno do aborto provocado em nível nacional ${ }^{3,4}$. O segundo tipo constitui-se de pesquisas à beira do leito, com mulheres internadas por complicações do aborto, nas quais as histórias de aborto são recuperadas por profissionais de saúderesponsáveis pelo atendimento médico. Grande parte dos estudos brasileiros sobre aborto foi assim realizada e apresenta dados de hospitais específicos ou determinadas localidades ${ }^{5-8} .0$ terceiro tipo combina novas técnicas de coleta da informação, mas procura pesquisar o fenômeno do aborto provocado também fora do ambiente hospitalar, tais como fizeram as sondagens com uso de técnica ao azar etécnica de urna9-12. Assim como as pesquisas à beira do leito, as pesquisas com técnicas de sigilo foram realizadas em localidades específicas.

As estimativas da magnitude do aborto divergem conformeas técnicas efontes utilizadas ${ }^{13}$. 15. A aplicação de distintos métodos de coleta de informação sobre aborto, utilizando questionários em diferentes amostras de população, levou a estimativas variadas. De acordo com o estudo de Lara e colaboradores, realizado no M éxico, a técnica de resposta ao azar encontra taxas de aborto mais elevadas, muito embora apresente as desvantagens de provocar erros de interpretação entre mulheres de baixa escolaridade exigir amostras muito maiores ${ }^{13}$. Os questionários autoadministrados al cançam taxas menores, mas são a técnica mais recomendada quando se pretende obter outras informações das entrevistadas. Em seguida, vêm as entrevistas por computador autoadministradas e as entrevistas face a face. No Brasil, Silva, após estudar um bairro da cidade deSão Paulo, concluiu queentrevistas face a face levavam a uma omissão de respostas sobre aborto de oitenta em cada cem mulheres entrevistadas ${ }^{12}$.

Atéo momento, as estimativas de aborto para todo o Brasil baseiam-se em técnicas indiretas. Um estudo baseado em internações associadas ao aborto em serviços públicos de saúde, por exemplo, calcula que houve 2,07 abortos para cada 100 mulheres entre 15 e 49 anos em 20053. Todavia, estimativas dessetipo dependem de um parâmetro - a razão de internações por aborto - que neste caso foi estabelecido assumindo-seque $20 \%$ dos abortos resultaram em internação registrada pelo Sistema de Internações H ospital ares do SUS. As técnicas diretas de estimação não dependem dessemultiplicador e podem, na verdade, contribuir para determiná-lo mais corretamente.

Estimativas diretas foram realizadas, mas para áreas geográficas menores. Um inquérito aplicou com sucesso a técnica de urna em uma amostra de domicílios na cidade de Pelotas (RS). A técnica de urna consiste em um questionário sigiloso preenchido pelas entrevistadas e depositado em uma urna, sendo indicada como uma das melhores alternativas para esse tipo de estimativa. 0 estudo de Pelotas identificou a indução de aborto em $7,2 \%$ das mulheres de todas as idades entrevistadas, quase o dobro do valor obtido quando a técnica de levantamento foi a das questões indiretas ${ }^{10}$. No grupo das mulheres que concluíram sua vida reprodutiva ( 45 a 49 anos), a estimativa édeque $12 \%$ delas induziram 0 aborto ao longo da vida.

Esse valor do estudo de Pelotas guarda alguma semel hança com outros realizados na Colômbia e no M éxico. Um inquérito nacional aplicando a técnica deurna em 1992 encontrou que22,9\% das mulheres urbanas de 15 a 55 anos fizeram aborto na Colômbia ${ }^{16}$. Em pesquisa na Cidade do M éxico e na área rural de Chiapas (M éxico), Lara e colaboradores estimam que, em 2001, $21,7 \%$ das mulheres de 15 a 55 anos entrevistadas já haviam tentado induzir o aborto ao menos uma vez na vida ${ }^{13}$. Posteriormente, utilizando a técnica de resposta ao azar em uma amostra de todo o país, os autores estimaram uma incidência total de aborto induzido no M éxico de $16 \%{ }^{17}$.

Este artigo apresenta os primeiros resultados da Pesquisa Nacional deAborto (PNA). A PNA é 
um levantamento por amostragem aleatória estratificada de domicílios que combinou duas técnicas de sondagem: a técnica de urna e questionários preenchidos por entrevistadoras. Sua cobertura abrangeu mulheres com idades entre 18 e 39 anos em 2010, em todo o Brasil urbano. 0 objetivo da PNA é oferecer dados sobre aborto no Brasil, a fim de subsidiar ações de saúde pública para as mulheres em idade reprodutiva e fornecer informações necessárias para o desenho de novas sondagens do tipo e parâmetros para estimativas indiretas.

\section{M etodologia}

Os dados foram levantados junto a mulheres adultas, combinando duas técnicas sequenciais. Primeiro, questionários autopreenchidos depositados em urna, procedimento conhecido como técnica de urna (ballot box technique), com perguntas fechadas sobre aborto. Segundo, questionários face a face preenchidos por entrevistadoras, que indagavam sobre informações sociais e demográficas. 0 objetivo da urna é enfatizar, no momento da entrevista, o sigilo das respostas, a fim de melhorar a fidedignidade de respostas a questões social mente controversas. A técnica, utilizada com sucesso em levantamentos anteriores sobreaborto ${ }^{1,10,13}$, foi inicialmentedesenhada para pesquisas deintenção de voto ${ }^{18}$ eestudosindicam que seu uso reduz drasticamente a incidência de não resposta a certas questões ${ }^{19,20}$. A urna e 0 questionário apresentavam textos e indicações gráficas que enfatizavam o sigilo das respostas, e a introdução oral às entrevistas deliberadamente reforçava seu caráter confidencial.

A metodologia e os questionários de urna foram validados em um procedimento de dez etapas, que incluíram três rodadas de avaliação por equipes externas de especialistas e dois testes de campo em que se aplicaram técnicas mistas sequenciais de entrevista (questionário e roteiro estruturado) em amostras aleatórias de domicílios. Ao todo, sete modelos de questionários foram avaliados para a produção da versão final do questionário de urna. Os procedimentos de validação dos instrumentos de pesquisa serão apresentados em detalhe em outra publicação.

0 universo amostrado pela pesquisa constitui-se de mulheres com idades entre 18 e 39 anos alfabetizadas e residentes no Brasil urbano em 2010. A idade mínima de 18 anos assegurava maioridade legal às respondentes e a máxima de 39 anos buscava minimizar erros de memória, um problema importante no caso de questões controversas ${ }^{21}$. Essa faixa etária compreende a maioria dos eventos reprodutivos das mulheres brasileiras. A alfabetização era um requisito inevitável para o tipo de questionário utilizado e a restrição a áreas urbanas buscava reduzir o viés associado à não realização de entrevistas por analfabetismo, cuja incidência entre mulheres acima de trinta anos é elevada em zonas rurais, segundo a Pesquisa Nacional por Amostra de Domicílios de $2008^{22}$.

0 inquérito foi realizado em uma amostra al eatória estratificada de domicílios com alocação proporcional à população de cada estrato. A amostra foi desenhada para um nível de confiança de $95 \%$, com erro amostral de dois pontos percentuais para os resultados nacionais. Os estratos da amostra foram compostos pelas unidades da Federação, sendo as capitais e os municípios de regiões metropolitanas incluídos com probabilidade igual a um e tamanho da amostra proporcional às suas populações. Dentro decada estrato, amostras probabilísticas de conglomerados foram selecionadas em três estágios: municípios com probabilidade proporcional ao tamanho (da população de mulheres alfabetizadas de 18 a 39 anos), setores censitários com probabilidade proporcional ao tamanho (da população de mulheres alfabetizadas de 18 a 39 anos) e mulheres em domicílios, utilizando cotas proporcionais deidade, condição de ocupação laboral egrau de instrução, resultando em uma amostra autoponderada de 2.002 entrevistas. Foi entrevistada apenas uma mulher por domicílio. A amostragem eas entrevistas foram realizadas pela Agência Ibope Inteligência, com o trabalho de campo ocorrendo entre 13 e 21 dejaneiro de 2010.

Cada entrevistada respondeu dois questionários, um autopreenchido edepositado na urna e outro aplicado por uma entrevistadora do sexo feminino ${ }^{23}$. Os dois questionários possuíam códigos que permitiam seu pareamento, mas não a identificação das participantes. 0 questionário da urna confirmava a idade exata e perguntava se a mulher havia realizado aborto; em caso afirmativo, com que idade foi o último aborto; se usou medicamento para fazêlo e se ficou internada em função desse aborto. 0 questionário aplicado pela entrevistadora continha questões sobre alfabetização, faixa etária, escolaridade, condição de ocupação laboral, frequência ao posto de saúde e ginecologista, situação conjugal, fecundidade, tamanho da família, religião e renda familiar. Seguindo recomendações para questionários autopreenchidos sobre saúde se- 
xual e reprodutiva em populações que incluem pessoas com baixa escolaridade, o formato do questionário da urna era simples, sem questões conceitualmente complexas ou envolvendo ordenamentos e respostas múltiplas ${ }^{24}$.

As perguntas foram realizadas em três etapas: questionário das entrevistadoras, questionário da urna e retorno ao questionário das entrevistadoras. A entrevista tinha início com questões sobre alfabetização, faixa etária, escolaridade e condição de ocupação laboral, que determinavam as cotas amostrais e, portanto, a seleção para participação na pesquisa. Em seguida, estavam questões de frequência ao posto de saúde e ginecologista, seguidas do questionário da urna. Finalmente, depois de depositado o questionário, concluía-se a entrevista com questões sobre situação conjugal, fecundidade, tamanho da família, religião e renda familiar apresentadas pelas entrevistadoras.

0 questionário da urna perguntava à mulher "Você já fez aborto alguma vez?". Além disso, a informação contextual do restante do questionário (três questões adicionais) enfatizava a sondagem do aborto induzido. M esmo assim, apesar da redação utilizada ter como objetivo a captação do aborto induzido, é possível que tenha captado também abortos espontâneos. Enunciados alternativos, mais incisivos na identificação da indução, foram avaliados nos pré-testes e descartados.

As estimativas referem-se a abortos feitos em qualquer período da vida das mulheres e não apenas àquel es ocorridos em 2010. As características sociodemográficas das mulheres são as observadas em 2010, mas são também apresentadas informações sobre o último aborto realizado. A escolha pelo último aborto teve como objetivo reduzir eventuais erros de memória.

A PNA foi financiada pelo Fundo $N$ acional de Saúde, como parte de uma investigação extensa sobre políticas de saúde reprodutiva no Brasil, e conduzida por duas instituições de pesquisa, a Universidade de Braślia e a Anis - Instituto deBioética, Direitos H umanos e Gênero. 0 projeto de pesquisa foi submetido ao Comitê deÉtica em Pesquisa do Instituto de Ciências Humanas da Universidade de Brasília e aprovado antes de sua execução, em conformidade com as diretrizes do Conselho Nacional de Saúde ${ }^{25}$. 0 termo de consentimento livre e esclarecido (TCLE) foi obtido oralmente e antes do início de cada abordagem às mulheres, como forma de garantir o sigilo e anonimato dos dados. Não houve registro de recusa quanto à aceitação do TCLE oral e as pesquisadoras de campo foram instruídas para o encaminhamento ao posto de saúde em caso de desconforto psicológico causado pela entrevista ou em caso de demandas por serviços de saúde reprodutiva. No entanto, não houve intercorrências dessa natureza.

\section{Resultados}

Há uma produção relativamente extensa sobreo tema do aborto no Brasil, que foi tratada por revisões recentes ${ }^{1,2}$. Todavia, dois fatores impedem a discussão dos resultados da PNA à luz dos achados de outras investigações sobre a magnitude do aborto no país. Primeiro, o campo écaracterizado por estudos de natureza local, sendo os estudos de abrangência nacional escassos. Segundo, a metodologia utilizada nas estimativas nacionais não permite comparação: os estudos abrangendo o Brasil estimam indiretamente a ocorrência de abortos em um período determinado, ao passo que a PN A estima diretamente mulheres que já fizeram aborto em al gum momento da vida. As diferenças nos períodos de referência - ano e ao longo da vida - e nos objetos-abortosemulheres quefizeram um ou mais abortos - fazem com que a comparação entre os resultados não seja possível.

Em 2010, no Brasil urbano, $15 \%$ das mulheres entrevistadas relataram ter realizado aborto al guma vez na vida (Tabela 1). O s resultados não se referem a números e proporções de abortos, mas sim a mulheres que fizeram aborto. Essas unidades de mensuração não são as mesmas, porque uma mulher pode abortar mais de uma vez ao longo da vida. 0 número de abortos é, seguramente, superior ao número de mulheres que fizeram aborto, mas os dados desta pesquisa não permitem estimar quanto. Além disso, o número total de abortos no país será maior do que o indicado nesteestudo seasáreas rurais ea população analfabeta forem também contabilizadas.

Assim como outros fatos cumulativos relacionados à vida reprodutiva, a proporção de mulheres que fizeram aborto cresce com a idade. Essa proporção varia de $6 \%$ para mulheres com idades entre 18 e 19 anos a $22 \%$ entremulheres de 35 a 39 anos. Isso mostra o quanto o aborto é um fenômeno comum na vida reprodutiva das muIheres. Em termos simples, isso significa que, ao final de sua vida reprodutiva, mais de um quinto das mulheres no Brasil urbano fez aborto.

Além da idade no momento da entrevista, a PNA perguntou a idade de realização do último 
aborto. As evidências do inquérito indicam que 0 aborto não éfeito apenas para retardar o início da vida reprodutiva ou evitar filhos em idades avançadas. $\mathrm{Na}$ verdade, cerca de $60 \%$ das muIheres fizeram seu último (ou único) aborto no centro do período reprodutivo, isto é, entre $18 \mathrm{e}$ 29 anos, sendo o pico da incidência entre 20 e 24 anos ( $24 \%$ nesta faixa etária apenas). Vale notar ainda que $15 \%$ das mulheres não sabem ou não responderam a idade com que realizaram o último aborto.

0 aborto é mais frequente entre mulheres de escolaridade muito baixa. A proporção de muIheres quefizeram aborto alcança $23 \%$ entreaque las com até o quarto ano do ensino fundamental (ou nível equivalente), ao passo que entre muIheres com o ensino médio concluído éde $12 \%$. É difícil, porém, imputar à escolarização um determinante direto do aborto por pelo menos duas razões: (1) os resultados refletem uma distribuição etária da educação na qual o nível de escolaridade é menor entre grupos que acumularam mais abortos ao longo do tempo, ou seja, as mulheres mais vel has e(2) porqueérazoável crer que os efeitos indiretos da educação sobreo aborto - afetando partici pação no mercado de trabaIho e salários, padrões de união conjugal, etc. sejam tão ou mais importantes que os efeitos diretos do nível de informação sobre reprodução e sexualidade que uma maior escolaridade seria capaz de acrescentar.

A incidência de aborto entre as mulheres de diferentes religiões é praticamenteigual. Não foi observada nenhuma diferença significativa entre grupos religiosos, estando boa parte das variações observadas dentro das margens de erro da pesquisa. Como a PNA reflete a composição religiosa das mulheres urbanas brasileiras, pouco menos de dois terços das mulheres que fizeram aborto são católicas, um quarto, protestantes ou evangélicas, e menos de um vigésimo, de outras religiões. Cerca de um décimo não respondeu ou não possui religião.

Como se observa na Tabela 2, cerca de metade das mulheres que fizeram aborto utilizaram algum tipo de medicamento para induzi-lo. Os abortosilegais real izados com medicamentostendem a ser mais seguros que os que utilizam outros meios, em particular quando o medicamento usado éo misoprostol, popularizado no Brasil na década de noventa ${ }^{26}$. A PNA não identifica quais os medicamentos utilizados pelas mulheres, mas é possível que entre eles o misoprostol ocupe uma posição importante. Se isso for verdade, os níveis de segurança do aborto não hospitalar no Brasil devem ser hoje superiores aos

Tabela 1. Realização de aborto segundo características sociais - mulheres de 18 a 39 anos, Brasil urbano, 2010.

\begin{tabular}{lcccc}
\hline \multicolumn{1}{c}{ Você já fez aborto al guma vez? } & N & Sim & $\%$ & C (95\%), pp. \\
\hline Todas as mulheres & 2.002 & 296 & $15 \%$ & 2 \\
Idade & & & & \\
18 e 19 & 191 & 11 & $6 \%$ & 1 \\
20 a 24 & 483 & 36 & $7 \%$ & 2 \\
25 a 29 & 488 & 84 & $17 \%$ & 2 \\
30 a 34 & 452 & 79 & $17 \%$ & 2 \\
35 a 39 & 388 & 86 & $22 \%$ & 2 \\
Escolaridade & & & & 2 \\
Até a 4a série do fundamental & 191 & 44 & $23 \%$ & 1 \\
5 a a 8a série do fundamental & 429 & 80 & $19 \%$ & 2 \\
Ensino médio & 974 & 115 & $12 \%$ & \\
Ensino superior & 408 & 57 & $14 \%$ & 2 \\
Religião & & & & 1 \\
Católica & 1.168 & 175 & $15 \%$ & 2 \\
Evangélica ou protestante & 551 & 72 & $13 \%$ & 2 \\
Outras religiões & 81 & 13 & $16 \%$ & $18 \%$ \\
Não tem religião/não respondeu & 202 & 36 & & \\
\hline
\end{tabular}

Fonte: Pesquisa Nacional de A borto, microdados da amostra, Brasil 2010. N ota: intervalos de confiança C a $95 \%$, em pontos percentuais (pp.) 


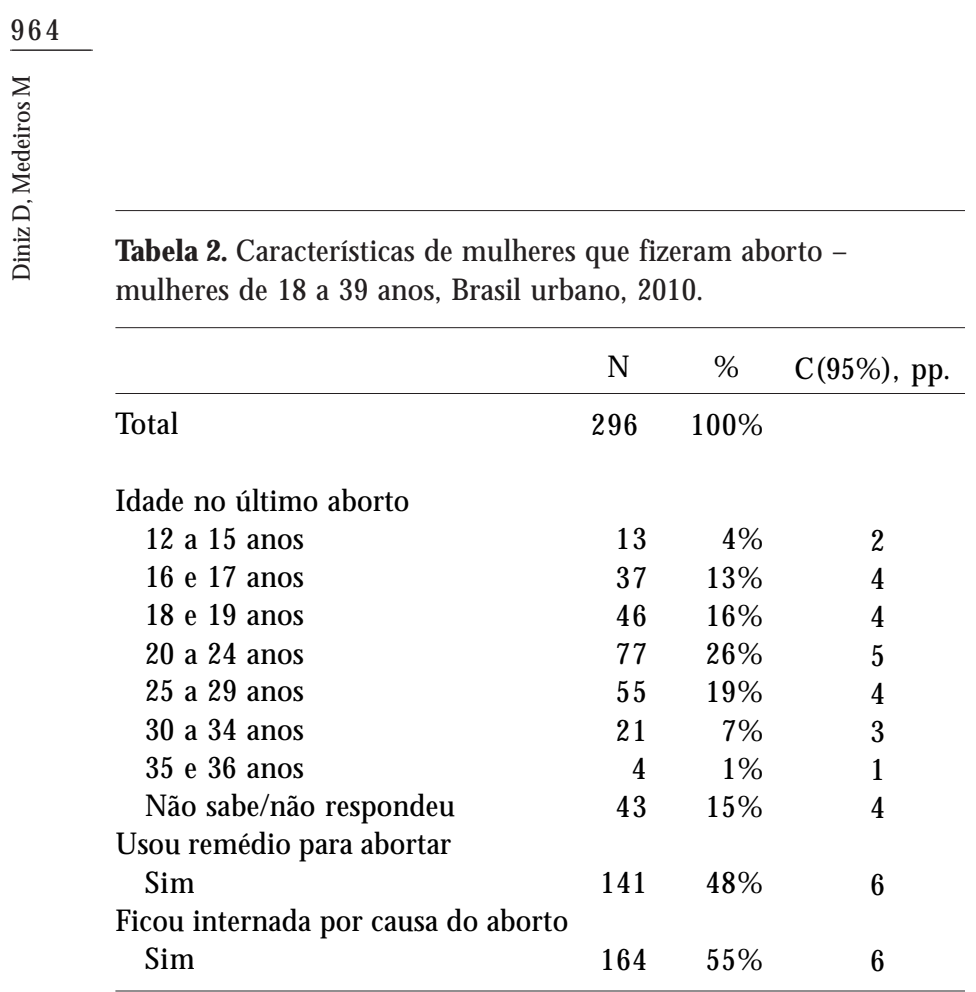

Fonte: Pesquisa N acional de Aborto, microdados da amostra, Brasil 2010. Nota: intervalos de confiança C a $95 \%$, em pontos percentuais (pp.)

observados em décadas passadas, uma hipótese que se fortalece com a queda da morbimortalidade por aborto inseguro no país $5^{1,2}$. Todavia, há ainda outra metade das mulheres que aborta sem recorrer a medicamentose, nos casos deindução, é possível que essas mulheres estejam abortando sob condições de saúde precárias, uma vez que grande parte delas tem baixo nível educacional.

Os níveis de internação pós-aborto são elevados e colocam o aborto como um problema de saúde pública no Brasil. Cerca de metade das mulheres que fizeram aborto recorreram ao sistema de saúde e foram internadas por complicações relacionadas ao aborto, o que corresponde a $8 \%$ das mulheres entrevistadas. Boa parte dessa internação poderia ter sido evitada se 0 aborto não fosse tratado como atividade clandestina e 0 acesso aos medicamentos seguros para aborto fosse garantido.

\section{Conclusões}

A Pesquisa Nacional deAborto (PNA) combinou duastécnicas desondagem, técnica deurna equestionários preenchidos por entrevistadoras, para levantar dados sobre aborto no Brasil urbano em uma amostra estratificada de 2.002 mulheres alfabetizadas com idades entre 18 e 39 anos em 2010. Seus resultados referem-se a mulheres que abortaram e não a abortos. 0 número de abortos no país é superior ao contabilizado pela pesquisa, não só porque uma mesma mulher pode abortar mais de uma vez, mas também porque as mulheres analfabetas e as áreas rurais do Brasil não foram cobertas pelo inquérito.

A PNA indica que o aborto é tão comum no Brasil que, ao completar quarenta anos, mais de uma em cada cinco mulheres já fez aborto. Tipicamente, 0 aborto é feito nas idades que compõem o centro do período reprodutivo feminino, isto é, entre 18 e 29 anos, e é mais comum entre mulheres de menor escolaridade, fato que pode estar relacionado a outras características sociais das mulheres de baixo nível educacional. A religião não éum fator importante para a diferenciação das mulheres no que diz respeito à realização do aborto. Refletindo a composição re ligiosa do país, a maioria dos abortos foi feita por católicas, seguidas de protestantes e evangélicas e, finalmente, por mulheres de outras religiões ou sem religião.

O uso de medicamentos para a indução do último aborto ocorreu em metade dos casos. Considerando que a maior parte das mulheres é de baixa escolaridade, é provável que para a outra metade das mulheres, que não fez uso de medicamentos, o aborto seja realizado em condições precárias de saúde. Não surpreende que os níveis de internação pós-aborto contabilizados pela PNA sejam elevados, ocorrendo em quase a metade dos casos. Um fenômeno tão comum e com consequências de saúde tão importantes coloca 0 aborto em posição de prioridade na agenda de saúde pública nacional. 


\section{Colaboradores}

D DinizeM M edeiros participaram conjuntamente de todas as etapas do estudo e são igualmente responsáveis pela redação do artigo.

\section{Agradecimentos}

Kátia Soares Braga (Anis - Instituto de Bioética, Direitos H umanose Gênero) foi responsável pelo levantamento internacional da literatura sobre técnicas de urna. Lívia Barbosa, Vanessa Carrião e Rosana Castro (Anis - Instituto de Bioética, Direitos Humanos e Gênero) foram responsáveis pela supervisão do trabal ho de campo e sistematização dos dados dos diários de campo. Flávia Farias (Fundação O swaldo Cruz) foi responsável pela diagramação final do questionário. A Agência Ibope Inteligência foi responsável pela execução da fase de pesquisa nacional. Agradecemos os comentários de Pedro Tauil, Ana M aria N ogales, Suzanne Serruya, Greice M enezes e M arilena Corrêa. $O$ financiamento do projeto I mplementação de Políticas de Atenção à Saúde da Mulher - Política Nacional dePlanejamento Familiar é da Diretoria Executiva do Fundo Nacional de Saúde, Ministério da Saúde do Brasil. O sautores agradecem às dezenove especialistas que revisaram os modelos de questionário, às vinte pesquisadoras de campo da fase de pré teste eàs 154 pessoas responsáveis pelo inquérito nacional, um grupo por demais extenso para ser nominado aqui.

\section{Referências}

1. Brasil. M inistério da Saúde. 20 anos de pesquisas sobre aborto no Brasil. Brasília: M inistério da Saúde; 2009.

2. M enezes $G$, Aquino E. Pesquisa sobre o aborto no Brasil: avanços e desafios para o campo da saúde coletiva. Cad Saude Publica 2009; 25(Supl. 2):S193S204.

3. Monteiro M, Adesse L. Estimativas de aborto induzido no Brasil e Grandes Regiões, 1992-2005. In: Anais do XV Encontro $\mathrm{N}$ acional de Estudos Populacionais; 2006; Caxambu. p. 1-10.

4. Singh S, Wulf D. Estimating abortion levels in Brazil, Colombia and Peru, using hospital admissions and fertility survey data. Int Fam Plan Perspect 1991;17(1):8-24.

5. Fonseca W, M isago C, Correia LL, Parente JAM, Oliveira FC. Determinantes do aborto provocado entre mulheres admitidas em hospitais em localidade da Região Nordeste do Brasil. Rev. Saude Publica 1996; 30(1):13-18.

6. Fonseca W, M isago C, Freitas P, Santos E, Fernandes L, Correia L. Características sócio-demográficas, reprodutivas e médicas de mulheres admitidas por aborto em hospital da Região Sul do Brasil. Cad Saude Publica 1998;14(2):279-286.

7. Lucena RCBD. Características de mulheres internadas por aborto em uma maternidade pública em Recife-PE: dimensão do problema e sua relação com a prática contraceptiva [dissertação]. Recife (PE): Centro de Pesquisas Aggeu Magalhães, Fundação Oswaldo Cruz; 2000.

8. Schor N. Investigação sobre ocorrência de aborto em pacientes de hospital de centro urbano do estado de São Paulo, Brasil. Rev. Saude Publica 1990; 24(2):144-151.

9. Olinto $M$. Estimativa da frequência de abortos induzidos: teste de uma metodologia. Rev Bras Estud Popul 1994;11:255-258.

10. Olinto M, M oreira Filho DC. Estimativa de aborto induzido: comparac'apo entre duas metodologias. Revista Panam Salud Publica 2004;15(5):331-336.

11. Silva R. Cegonhas indesejadas: aborto provocado. Estud Fem 1993;1(1):123-133.

12. Silva R. O uso da técnica de resposta ao azar (TRA) na caracterização do aborto ilegal. Rev Bras Estud Popul 1993;10(1/2):41-56.

13. Lara D, Strickler J, Olavarrieta CD, Ellertson C. Measuring induced abortion in M exico: a comparison of four methodologies. Sociol M ethods Res 2004; 32(4):529-558.

14. Barreto T. Investigating induced abortion in developing countries: methods and problems. Stud Fam Plan 1992;23(3):159-170.

15. Rossier C. Estimating induced abortion rates: a review. Stud Fam Plan 2003;34(2):87.

16. Zamudio L, Rubiano N, Wartenberg L. The incidence and social and demographic characteristics of abortion in Colombia. In: M undigo Al, Indriso $C$, editors. Abortion in the developing world. London; New York: Zen Books; 1999. p. 149-172. 
17. Lara D, Garcia SG, Ellertson C, Camlin C, Suarez J. The measure of induced abortion levels in Mexico using random response technique. Sociol M ethods Res 2006; 35(2):279-301.

18. Benson LE. Studies in secret-ballot technique. Public Opin Q 1941; 5(1):79-82.

19. Bishop GF, Fisher BS. "Secret ballots" and self-reports in an exit-poll experiment. Public O pin Q 1995; 59(4):568-588.

20. Diskin A, Felsenthal DS. Do they lie? Int Pol Sci Rev 1981; 2(4):407-422.

21. Bradley KA, Kivlahan DR, Bush KR, M cdonell MB, Finn SD. Variations on the CAGE alcohol screening questionnaire: strengths and limitations in VA general medical patients. Alcohol Clin Exp Res 2001; 25(10):1472-1478.

22. Instituto Brasileiro de Geografia e Estatística. Pesquisa Nacional por Amostra de Domićlios 2008 [CDROM ]. Rio de Janeiro: IBGE; 2008.

23. Catania JA, Binson D, Canchola J, Pollack LM, Hauck W, Coates TJ. Effects of interviewer gender, interviewer choice, and item wording on responses to questions concerning sexual behavior. Public 0 pin Q 1996; 60(3):345-375.

24. Plummer $M L$, Wight $D$, Ross $D A$, Balira R, Anêmona A, Todd J, Salamba Z, Obasi AI, Grosskurth H, Changalunga J, Hayes RJ. Asking semi-literate adolescents about sexual behaviour: the validity of assisted self-completion questionnaire (ASCQ) data in rural Tanzania. Trop M ed Int Health 2004;9(6):737754.

25. Brasil. Conselho Nacional de Saúde. Resolução 196/ 1996: diretrizes e normas regulamentadoras de pesquisas envolvendo seres humanos. Brasília: M inistério da Saúde; 1996.

26. Barbosa RM, Arilha MA. A experiência brasileira com o Cytotec. Estud Fem 1993;1(2):408-417.

Artigo apresentado em 24/03/2010

A provado em 11/05/2010

Versão final apresentada em 17/05/2010 